\title{
Measurement of the Absolute Branching Fraction of the Inclusive Semileptonic $\Lambda_{c}^{+}$Decay
}

M. Ablikim, ${ }^{1}$ M. N. Achasov, ${ }^{9, d}$ S. Ahmed, ${ }^{14}$ M. Albrecht, ${ }^{4}$ M. Alekseev, ${ }^{53 a, 53 c}$ A. Amoroso, ${ }^{53 a, 53 c}$ F. F. An, ${ }^{1}$ Q. An,${ }^{50,40}$ J. Z. Bai, ${ }^{1}$ Y. Bai, ${ }^{39}$ O. Bakina, ${ }^{24}$ R. Baldini Ferroli, ${ }^{20 a}$ Y. Ban, ${ }^{32}$ D. W. Bennett, ${ }^{19}$ J. V. Bennett, ${ }^{5}$ N. Berger, ${ }^{23}$ M. Bertani, ${ }^{20 a}$ D. Bettoni, ${ }^{21 a}$ J. M. Bian, ${ }^{47}$ F. Bianchi, ${ }^{53 a, 53 c}$ E. Boger, ${ }^{24, b}$ I. Boyko, ${ }^{24}$ R. A. Briere,${ }^{5}$ H. Cai, ${ }^{55}$ X. Cai,${ }^{1,40}$ O. Cakir, ${ }^{43 a}$ A. Calcaterra, ${ }^{20 a}$ G. F. Cao, ${ }^{1,44}$ S. A. Cetin, ${ }^{43 b}$ J. Chai, ${ }^{53 c}$ J. F. Chang, ${ }^{1,40}$ G. Chelkov, ${ }^{24, b, c}$ G. Chen, ${ }^{1}$ H. S. Chen, ${ }^{1,44}$ J. C. Chen, ${ }^{1}$ M. L. Chen, ${ }^{1,40}$ P. L. Chen, ${ }^{51}$ S. J. Chen, ${ }^{30}$ X. R. Chen, ${ }^{27}$ Y. B. Chen, ${ }^{1,40}$ X. K. Chu, ${ }^{32}$ G. Cibinetto, ${ }^{21 a}$ H. L. Dai, ${ }^{1,40}$ J. P. Dai, ${ }^{35, h}$ A. Dbeyssi $1,{ }^{14}$ D. Dedovich, ${ }^{24}$ Z. Y. Deng, ${ }^{1}$ A. Denig, ${ }^{23}$ I. Denysenko, ${ }^{24}$ M. Destefanis, ${ }^{53 a, 53 c}$ F. De Mori ${ }^{53 a, 53 c}$ Y. Ding, ${ }^{28}$ C. Dong, ${ }^{31}$ J. Dong, ${ }^{1,40}$ L. Y. Dong, ${ }^{1,44}$ M. Y. Dong,,${ }^{1,40,44}$ Z. L. Dou, ${ }^{30}$ S. X. Du, ${ }^{57}$ P. F. Duan, ${ }^{1}$ J. Fang, ${ }^{1,40}$ S. S. Fang, ${ }^{1,44}$ Y. Fang, ${ }^{1}$ R. Farinelli, ${ }^{21,21 b}$ L. Fava, ${ }^{5 b, 53 c}$ S. Fegan, ${ }^{23}$ F. Feldbauer, ${ }^{4}$ G. Felici, ${ }^{20 a}$ C. Q. Feng, ${ }^{50,40}$ E. Fioravanti, ${ }^{21 a}$ M. Fritsch, ${ }^{4}$ C. D. Fu, ${ }^{1}$ Q. Gao, ${ }^{1}$ X. L. Gao,${ }^{50,40}$ Y. Gao, ${ }^{42}$ Y. G. Gao, ${ }^{6}$ Z. Gao, ${ }^{50,40}$ B. Garillon, ${ }^{23}$ I. Garzia, ${ }^{21 a}$ K. Goetzen, ${ }^{10}$ L. Gong, ${ }^{31}$ W. X. Gong, ${ }^{1,40}$ W. Gradl, ${ }^{23}$ M. Greco, ${ }^{53 a, 53 c}$ M. H. Gu, ${ }^{1,40}$ Y. T. Gu, ${ }^{12}$ A. Q. Guo, ${ }^{1}$ R. P. Guo ${ }^{1,44}$ Y. P. Guo, ${ }^{23}$ Z. Haddadi, ${ }^{26}$ S. Han,${ }^{55}$ X. Q. Hao ${ }^{15}$ F. A. Harris, ${ }^{45}$ K. L. He,${ }^{1,44}$ X. Q. He ${ }^{49}$ F. H. Heinsius,${ }^{4}$ T. Held, ${ }^{4}$ Y. K. Heng,,${ }^{1,40,44}$ T. Holtmann, ${ }^{4}$ Z. L. Hou, ${ }^{1}$ H. M. Hu, ${ }^{1,44}$ J. F. Hu, ${ }^{35, h}$ T. Hu, ${ }^{1,40,44}$ Y. Hu, ${ }^{1}$ G. S. Huang, ${ }^{50,40}$ J. S. Huang, ${ }^{15}$ X. T. Huang, ${ }^{34}$ X. Z. Huang, ${ }^{30}$ Z. L. Huang,${ }^{28}$ T. Hussain,${ }^{52}$ W. Ikegami Andersson, ${ }^{54}$ Q. Ji, ${ }^{1}$ Q. P. Ji, ${ }^{15}$ X. B. Ji, ${ }^{1,44}$ X. L. Ji, ${ }^{1,40}$ X. S. Jiang,,${ }^{1,40,44}$ X. Y. Jiang, ${ }^{31}$ J. B. Jiao, ${ }^{34}$ Z. Jiao, ${ }^{17}$ D. P. Jin, ${ }^{1,40,44}$ S. Jin, ${ }^{1,44}$ Y. Jin, ${ }^{46}$ T. Johansson, ${ }^{54}$ A. Julin, ${ }^{47}$ N. Kalantar-Nayestanaki, ${ }^{26}$ X. L. Kang, ${ }^{1}$ X. S. Kang, ${ }^{31}$ M. Kavatsyuk, ${ }^{26}$ B. C. Ke, ${ }^{5}$ T. Khan,${ }^{50,40}$ A. Khoukaz, ${ }^{48}$ P. Kiese, ${ }^{23}$ R. Kliemt, ${ }^{10}$ L. Koch, ${ }^{25}$ O. B. Kolcu, ${ }^{43 b, f}$ B. Kopf, ${ }^{4}$ M. Kornicer, ${ }^{45}$ M. Kuemmel, ${ }^{4}$ M. Kuessner, ${ }^{4}$ M. Kuhlmann, ${ }^{4}$ A. Kupsc ${ }^{54}$ W. Kühn, ${ }^{25}$ J. S. Lange, ${ }^{25}$ M. Lara, ${ }^{19}$ P. Larin, ${ }^{14}$ L. Lavezzi, ${ }^{53 c}$ H. Leithoff, ${ }^{23}$ C. Leng, ${ }^{53 c}$ C. Li,${ }^{54}$ Cheng Li ${ }^{50,40}$ D. M. Li, ${ }^{57}$ F. Li, ${ }^{1,40}$ F. Y. Li, ${ }^{32}$ G. Li, ${ }^{1}$ H. B. Li ${ }^{1,44}$ H. J. Li, ${ }^{1,44}$ J. C. Li ${ }^{1}{ }^{1}{ }^{4} \mathrm{Li},{ }^{33}$ K. J. Li, ${ }^{41} \mathrm{Kang} \mathrm{Li}^{13}{ }^{13} \mathrm{Ke} \mathrm{Li}^{34}$ Lei $\mathrm{Li}^{3}{ }^{3}$ P. L. Li ${ }^{50,40}$ P. R. Li ${ }^{44,7}$ Q. Y. Li, ${ }^{34}$ W. D. Li, ${ }^{1,44}$ W. G. Li, ${ }^{1}$ X. L. Li ${ }^{34}$ X. N. Li, ${ }^{1,40}$ X. Q. Li, ${ }^{31}$ Z. B. Li ${ }^{41}$ H. Liang, ${ }^{50,40}$ Y. F. Liang, ${ }^{37}$ Y. T. Liang, ${ }^{25}$ G. R. Liao, ${ }^{11}$ D. X. Lin, ${ }^{14}$ B. Liu, ${ }^{35, h}$ B. J. Liu, ${ }^{1}$ C. X. Liu, ${ }^{1}$ D. Liu, ${ }^{50,40}$ F. H. Liu, ${ }^{36}$ Fang Liu, ${ }^{1}$ Feng Liu, ${ }^{6}$ H. B. Liu, ${ }^{12}$ H. M. Liu, ${ }^{1,44}$ Huanhuan Liu, ${ }^{1}$ Huihui Liu, ${ }^{16}$ J. B. Liu, ${ }^{50,40}$ J. Y. Liu, ${ }^{1,44}$ K. Liu, ${ }^{42}$ K. Y. Liu, ${ }^{28}$ Ke Liu, ${ }^{6}$ L. D. Liu, ${ }^{32}$ P. L. Liu,,${ }^{1,40}$ Q. Liu, ${ }^{44}$ S. B. Liu,${ }^{50,40}$ X. Liu, ${ }^{27}$ Y. B. Liu, ${ }^{31}$ Z. A. Liu,,${ }^{1,40,44}$ Zhiqing Liu, ${ }^{23}$

Y. F. Long, ${ }^{32}$ X. C. Lou, ${ }^{1,40,44}$ H. J. Lu, ${ }^{17}$ J. G. Lu, ${ }^{1,40}$ Y. Lu, ${ }^{1}$ Y. P. Lu, ${ }^{1,40}$ C. L. Luo, ${ }^{29}$ M. X. Luo, ${ }^{56}$ X. L. Luo, ${ }^{1,40}$

X. R. Lyu, ${ }^{44}$ F. C. Ma,${ }^{28}$ H. L. Ma, ${ }^{1}$ L. L. Ma,${ }^{34}$ M. M. Ma, ${ }^{1,44}$ Q. M. Ma, ${ }^{1}$ T. Ma,,${ }^{1}$ X. N. Ma ${ }^{31}$ X. Y. Ma, ${ }^{1,40}$ Y. M. Ma, ${ }^{34}$ F. E. Maas, ${ }^{14}$ M. Maggiora, ${ }^{53 a, 53 c}$ Q. A. Malik, ${ }^{52}$ Y. J. Mao, ${ }^{32}$ Z. P. Mao, ${ }^{1}$ S. Marcello, ${ }^{53 a, 53 c}$ Z. X. Meng, ${ }^{46}$

J. G. Messchendorp, ${ }^{26}$ G. Mezzadri, ${ }^{21 b}$ J. Min, ${ }^{1,40}$ T. J. Min, ${ }^{1}$ R. E. Mitchell, ${ }^{19}$ X. H. Mo, ${ }^{1,40,44}$ Y. J. Mo, ${ }^{6}$

C. Morales Morales, ${ }^{14}$ N. Yu. Muchnoi ${ }^{9, d}$ H. Muramatsu, ${ }^{47}$ A. Mustafa, ${ }^{4}$ Y. Nefedov,${ }^{24}$ F. Nerling, ${ }^{10}$ I. B. Nikolaev, ${ }^{9, d}$ Z. Ning, ${ }^{1,40}$ S. Nisar, ${ }^{8}$ S. L. Niu, ${ }^{1,40}$ X. Y. Niu, ${ }^{1,44}$ S. L. Olsen, ${ }^{33, j}$ Q. Ouyang,,${ }^{1,40,44}$ S. Pacetti, ${ }^{20 b}$ Y. Pan,${ }^{50,40}$

M. Papenbrock, ${ }^{54}$ P. Patteri, ${ }^{20 a}$ M. Pelizaeus, ${ }^{4}$ J. Pellegrino, ${ }^{53 a, 53 c}$ H. P. Peng, ${ }^{50,40}$ K. Peters, ${ }^{10, \mathrm{~g}}$ J. Pettersson, ${ }^{54}$ J. L. Ping, ${ }^{29}$ R. G. Ping, ${ }^{1,44}$ A. Pitka, ${ }^{4}$ R. Poling, ${ }^{47}$ V. Prasad,${ }^{50,40}$ H. R. Qi, ${ }^{2}$ M. Qi ${ }^{30}$ T. Y. Qi, ${ }^{2}$ S. Qian, ${ }^{1,40}$ C. F. Qiao, ${ }^{44}$ N. Qin, ${ }^{55}$ X. S. Qin, ${ }^{4}$ Z. H. Qin, ${ }^{1,40}$ J. F. Qiu, ${ }^{1}$ K. H. Rashid, ${ }^{52, i}$ C. F. Redmer, ${ }^{23}$ M. Richter, ${ }^{4}$ M. Ripka, ${ }^{23}$ M. Rolo,${ }^{53 c}$ G. Rong, ${ }^{1,44}$ Ch. Rosner, ${ }^{14}$ A. Sarantsev, ${ }^{24, e}$ M. Savrié, ${ }^{21 b}$ C. Schnier, ${ }^{4}$ K. Schoenning, ${ }^{54}$ W. Shan, ${ }^{32}$ M. Shao, ${ }^{50,40}$ C. P. Shen, ${ }^{2}$

P. X. Shen, ${ }^{31}$ X. Y. Shen, ${ }^{1,44}$ H. Y. Sheng, ${ }^{1}$ X. Shi, ${ }^{1,40}$ J. J. Song,${ }^{34}$ W. M. Song, ${ }^{34}$ X. Y. Song, ${ }^{1}$ S. Sosio, ${ }^{53 a, 53 c}$ C. Sowa, ${ }^{4}$ S. Spataro, ${ }^{53 a, 53 c}$ G. X. Sun, ${ }^{1}$ J. F. Sun, ${ }^{15}$ L. Sun, ${ }^{55}$ S. S. Sun, ${ }^{1,44}$ X. H. Sun, ${ }^{1}$ Y. J. Sun, ${ }^{50,40}$ Y. K. Sun, ${ }^{50,40}$ Y.Z. Sun, ${ }^{1}$ Z. J. Sun, ${ }^{1,40}$ Z. T. Sun, ${ }^{19}$ C. J. Tang, ${ }^{37}$ G. Y. Tang, ${ }^{1}$ X. Tang, ${ }^{1}$ I. Tapan, ${ }^{43 \mathrm{c}}$ M. Tiemens, ${ }^{26}$ B. Tsednee, ${ }^{22}$ I. Uman, ${ }^{43 d}$ G. S. Varner, ${ }^{45}$ B. Wang, ${ }^{1}$ B. L. Wang, ${ }^{44}$ D. Wang, ${ }^{32}$ D. Y. Wang, ${ }^{32}$ Dan Wang,${ }^{44}$ K. Wang, ${ }^{1,40}$ L. L. Wang, ${ }^{1}$ L. S. Wang, ${ }^{1}$

M. Wang, ${ }^{34}$ Meng Wang, ${ }^{1,44}$ P. Wang, ${ }^{1}$ P. L. Wang, ${ }^{1}$ W. P. Wang, ${ }^{50,40}$ X. F. Wang, ${ }^{42}$ Y. Wang, ${ }^{38}$ Y. D. Wang, ${ }^{14}$

Y. F. Wang, ${ }^{1,40,44}$ Y. Q. Wang, ${ }^{23}$ Z. Wang, ${ }^{1,40}$ Z. G. Wang, ${ }^{1,40}$ Z. Y. Wang, ${ }^{1}$ Zongyuan Wang, ${ }^{1,44}$ T. Weber, ${ }^{4}$ D. H. Wei, ${ }^{11}$ P. Weidenkaff, ${ }^{23}$ S. P. Wen, ${ }^{1}$ U. Wiedner, ${ }^{4}$ M. Wolke, ${ }^{54}$ L. H. Wu, ${ }^{1}$ L. J. Wu, ${ }^{1,44}$ Z. Wu, ${ }^{1,40}$ L. Xia, ${ }^{50,40}$ Y. Xia, ${ }^{18}$ D. Xiao, ${ }^{1}$ H. Xiao, ${ }^{51}$ Y. J. Xiao, ${ }^{1,44}$ Z. J. Xiao, ${ }^{29}$ Y. G. Xie ${ }^{1,40}$ Y. H. Xie, ${ }^{6}$ X. A. Xiong, ${ }^{1,44}$ Q. L. Xiu, ${ }^{1,40}$ G. F. Xu, ${ }^{1}$ J. J. Xu, ${ }^{1,44}$ L. Xu, ${ }^{1}$ Q. J. Xu, ${ }^{13}$ Q. N. Xu, ${ }^{44}$ X. P. Xu ${ }^{38}$ L. Yan, ${ }^{53 a, 53 c}$ W. B. Yan, ${ }^{50,40}$ W. C. Yan, ${ }^{2}$ Y. H. Yan, ${ }^{18}$ H. J. Yang, ${ }^{35, h}$ H. X. Yang, ${ }^{1}$ L. Yang, ${ }^{55}$ Y. H. Yang, ${ }^{30}$ Y. X. Yang, ${ }^{11}$ M. Ye, ${ }^{1,40}$ M. H. Ye, ${ }^{7}$ J. H. Yin, ${ }^{1}$ Z. Y. You, ${ }^{41}$ B. X. Yu, ${ }^{1,40,44}$ C. X. Yu, ${ }^{31}$ J. S. Yu, ${ }^{27}$ C. Z. Yuan, ${ }^{1,44}$ Y. Yuan, ${ }^{1}$ A. Yuncu, ${ }^{43 b, a}$ A. A. Zafar ${ }^{52}$ Y. Zeng, ${ }^{18}$ Z. Zeng ${ }^{50,40}$ B. X. Zhang, ${ }^{1}$ B. Y. Zhang, ${ }^{1,40}$ C. C. Zhang, ${ }^{1}$ D. H. Zhang, ${ }^{1}$ H. H. Zhang, ${ }^{41}$ H. Y. Zhang, ${ }^{1,40}$ J. Zhang, ${ }^{1,44}$ J. L. Zhang, ${ }^{1}$ J. Q. Zhang, ${ }^{4}$ J. W. Zhang, ${ }^{1,40,44}$ J. Y. Zhang, ${ }^{1}$ J. Z. Zhang, ${ }^{1,44}$ K. Zhang, ${ }^{1,44}$ L. Zhang, ${ }^{42}$ S. Q. Zhang, ${ }^{31}$ X. Y. Zhang, ${ }^{34}$ Y. H. Zhang, ${ }^{1,40}$ Y. T. Zhang, ${ }^{50,40}$ Yang Zhang, ${ }^{1}$ Yao Zhang, ${ }_{1}^{1}$ Yu Zhang, ${ }^{44}$ Z. H. Zhang, ${ }^{6}$ Z. P. Zhang, ${ }^{50}$ Z. Y. Zhang, ${ }^{55}$ G. Zhao, ${ }^{1}$ J. W. Zhao, ${ }^{1,40}$ J. Y. Zhao, ${ }^{1,44}$ J. Z. Zhao, ${ }^{1,40}$

Lei Zhao, ${ }^{50,40}$ Ling Zhao, ${ }^{1}$ M. G. Zhao, ${ }^{31}$ Q. Zhao, ${ }^{1}$ S. J. Zhao, ${ }^{57}$ T. C. Zhao, ${ }^{1}$ Y. B. Zhao, ${ }^{1,40}$ Z. G. Zhao, ${ }^{50,40}$ 
A. Zhemchugov, ${ }^{24, b}$ B. Zheng, ${ }^{51}$ J. P. Zheng, ${ }^{1,40}$ Y. H. Zheng, ${ }^{44}$ B. Zhong, ${ }^{29}$ L. Zhou, ${ }^{1,40}$ X. Zhou, ${ }^{55}$ X. K. Zhou, ${ }^{50,40}$ X. R. Zhou, ${ }^{50,40}$ X. Y. Zhou, ${ }^{1}$ J. Zhu, ${ }^{31}$ J. Zhu, ${ }^{41}$ K. Zhu, ${ }^{1}$ K. J. Zhu ${ }^{1,40,44}$ S. Zhu, ${ }^{1}$ S. H. Zhu, ${ }^{49}$ X. L. Zhu, ${ }^{42}$ Y. C. Zhu, ${ }^{50,40}$ Y. S. Zhu, ${ }^{1,44}$ Z. A. Zhu, ${ }^{1,44}$ J. Zhuang, ${ }^{1,40}$ B. S. Zou, ${ }^{1}$ and J. H. Zou ${ }^{1}$

(BESIII Collaboration)

\author{
${ }^{1}$ Institute of High Energy Physics, Beijing 100049, People's Republic of China \\ ${ }^{2}$ Beihang University, Beijing 100191, People's Republic of China \\ ${ }^{3}$ Beijing Institute of Petrochemical Technology, Beijing 102617, People's Republic of China \\ ${ }^{4}$ Bochum Ruhr-University, D-44780 Bochum, Germany \\ ${ }^{5}$ Carnegie Mellon University, Pittsburgh, Pennsylvania 15213, USA \\ ${ }^{6}$ Central China Normal University, Wuhan 430079, People's Republic of China \\ ${ }^{7}$ China Center of Advanced Science and Technology, Beijing 100190, People's Republic of China \\ ${ }^{8}$ COMSATS Institute of Information Technology, Lahore, Defence Road, Off Raiwind Road, 54000 Lahore, Pakistan \\ ${ }^{9}$ G.I. Budker Institute of Nuclear Physics SB RAS (BINP), Novosibirsk 630090, Russia \\ ${ }^{10}$ GSI Helmholtzcentre for Heavy Ion Research GmbH, D-64291 Darmstadt, Germany \\ ${ }^{11}$ Guangxi Normal University, Guilin 541004, People's Republic of China \\ ${ }^{12}$ Guangxi University, Nanning 530004, People's Republic of China \\ ${ }^{13}$ Hangzhou Normal University, Hangzhou 310036, People's Republic of China \\ ${ }^{14}$ Helmholtz Institute Mainz, Johann-Joachim-Becher-Weg 45, D-55099 Mainz, Germany \\ ${ }^{15}$ Henan Normal University, Xinxiang 453007, People's Republic of China \\ ${ }^{16}$ Henan University of Science and Technology, Luoyang 471003, People's Republic of China \\ ${ }^{17}$ Huangshan College, Huangshan 245000, People's Republic of China \\ ${ }^{18}$ Hunan University, Changsha 410082, People's Republic of China \\ ${ }^{19}$ Indiana University, Bloomington, Indiana 47405, USA \\ ${ }^{20 a}$ INFN Laboratori Nazionali di Frascati, I-00044, Frascati, Italy \\ ${ }^{20 \mathrm{~b}}$ INFN and University of Perugia, I-06100, Perugia, Italy \\ ${ }^{21 \mathrm{a}}$ INFN Sezione di Ferrara, I-44122, Ferrara, Italy \\ ${ }^{21 b}$ University of Ferrara, I-44122, Ferrara, Italy \\ ${ }^{22}$ Institute of Physics and Technology, Peace Ave. 54B, Ulaanbaatar 13330, Mongolia \\ ${ }^{23}$ Johannes Gutenberg University of Mainz, Johann-Joachim-Becher-Weg 45, D-55099 Mainz, Germany \\ ${ }^{24}$ Joint Institute for Nuclear Research, 141980 Dubna, Moscow region, Russia \\ ${ }^{25}$ Justus-Liebig-Universitaet Giessen, II. Physikalisches Institut, Heinrich-Buff-Ring 16, D-35392 Giessen, Germany \\ ${ }^{26}$ KVI-CART, University of Groningen, NL-9747 AA Groningen, Netherlands \\ ${ }^{27}$ Lanzhou University, Lanzhou 730000, People's Republic of China \\ ${ }^{28}$ Liaoning University, Shenyang 110036, People's Republic of China \\ ${ }^{29}$ Nanjing Normal University, Nanjing 210023, People's Republic of China \\ ${ }^{30}$ Nanjing University, Nanjing 210093, People's Republic of China \\ ${ }^{31}$ Nankai University, Tianjin 300071, People's Republic of China \\ ${ }^{32}$ Peking University, Beijing 100871, People's Republic of China \\ ${ }^{33}$ Seoul National University, Seoul, 151-747 Korea \\ ${ }^{34}$ Shandong University, Jinan 250100, People's Republic of China \\ ${ }^{35}$ Shanghai Jiao Tong University, Shanghai 200240, People's Republic of China \\ ${ }^{36}$ Shanxi University, Taiyuan 030006, People's Republic of China \\ ${ }^{37}$ Sichuan University, Chengdu 610064, People's Republic of China \\ ${ }^{38}$ Soochow University, Suzhou 215006, People's Republic of China \\ ${ }^{39}$ Southeast University, Nanjing 211100, People's Republic of China \\ ${ }^{40}$ State Key Laboratory of Particle Detection and Electronics, Beijing 100049, Hefei 230026, People's Republic of China \\ ${ }^{41}$ Sun Yat-Sen University, Guangzhou 510275, People's Republic of China \\ ${ }^{42}$ Tsinghua University, Beijing 100084, People's Republic of China \\ ${ }^{43 a}$ Ankara University, 06100 Tandogan, Ankara, Turkey \\ ${ }^{43 \mathrm{~b}}$ Istanbul Bilgi University, 34060 Eyup, Istanbul, Turkey \\ ${ }^{43 c}$ Uludag University, 16059 Bursa, Turkey \\ ${ }^{43 \mathrm{~d}}$ Near East University, Nicosia, North Cyprus, Mersin 10, Turkey \\ ${ }^{44}$ University of Chinese Academy of Sciences, Beijing 100049, People's Republic of China \\ ${ }^{45}$ University of Hawaii, Honolulu, Hawaii 96822, USA \\ ${ }^{46}$ University of Jinan, Jinan 250022, People's Republic of China \\ ${ }^{47}$ University of Minnesota, Minneapolis, Minnesota 55455, USA \\ ${ }^{48}$ University of Muenster, Wilhelm-Klemm-Str. 9, 48149 Muenster, Germany
}




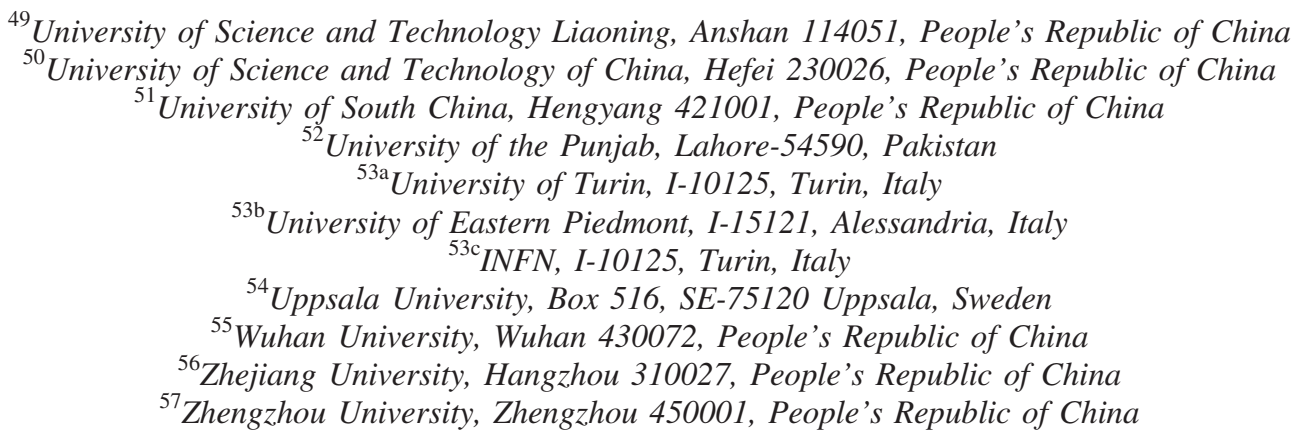

(Received 25 May 2018; published 18 December 2018)

\begin{abstract}
Using a data sample of $e^{+} e^{-}$collisions corresponding to an integrated luminosity of $567 \mathrm{pb}^{-1}$ collected at a center-of-mass energy of $\sqrt{s}=4.6 \mathrm{GeV}$ with the BESIII detector, we measure the absolute branching fraction of the inclusive semileptonic $\Lambda_{c}^{+}$decay with a double-tag method. We obtain $\mathcal{B}\left(\Lambda_{c}^{+} \rightarrow X e^{+} \nu_{e}\right)=$ $(3.95 \pm 0.34 \pm 0.09) \%$, where the first uncertainty is statistical and the second systematic. Using the known $\Lambda_{c}^{+}$lifetime and the charge-averaged semileptonic decay width of nonstrange charmed mesons $\left(D^{0}\right.$ and $\left.D^{+}\right)$, we obtain the ratio of the inclusive semileptonic decay widths $\Gamma\left(\Lambda_{c}^{+} \rightarrow X e^{+} \nu_{e}\right) /$ $\bar{\Gamma}\left(D \rightarrow X e^{+} \nu_{e}\right)=1.26 \pm 0.12$.
\end{abstract}

DOI: 10.1103/PhysRevLett.121.251801

Since the first observation of the $\Lambda_{c}^{+}$baryon, the lightest baryon containing a charm quark, in 1979 [1], its hadronic decays have been studied extensively. However, information about semileptonic decays of the $\Lambda_{c}^{+}$baryon is sparse [2-6]. The measurement of the branching fraction of $\Lambda_{c}^{+} \rightarrow$ $\Lambda l^{+} \nu_{l}(l=e, \mu)$ was first performed by the ARGUS collaboration [3] and then by the CLEO collaboration [4] before 1994. Recently, the BESIII collaboration measured the absolute branching fraction of $\Lambda_{c}^{+} \rightarrow \Lambda e^{+} \nu_{e}$ and $\Lambda_{c}^{+} \rightarrow$ $\Lambda \mu^{+} \nu_{\mu}$ to be $(3.63 \pm 0.43) \%$ [5] and $(3.49 \pm 0.53) \%$ [6], respectively. A comparison of the exclusive semileptonic decay branching fraction $\mathcal{B}\left(\Lambda_{c}^{+} \rightarrow \Lambda e^{+} \nu_{e}\right)$ and the inclusive semileptonic decay branching fraction $\mathcal{B}\left(\Lambda_{c}^{+} \rightarrow X e^{+} \nu_{e}\right)$, where $X$ refers to any possible particle system, will guide searches for new semileptonic decay modes. In addition, using the known $\Lambda_{c}^{+}$lifetime, the semileptonic decay width $\Gamma\left(\Lambda_{c}^{+} \rightarrow X e^{+} \nu_{e}\right)$ can be determined. Comparing $\Gamma\left(\Lambda_{c}^{+} \rightarrow\right.$ $\left.X e^{+} \nu_{e}\right)$ with the charge-averaged nonstrange $D$ semileptonic decay width $\bar{\Gamma}\left(D \rightarrow X e^{+} \nu_{e}\right)$, the ratio $\left[\Gamma\left(\Lambda_{c}^{+} \rightarrow X e^{+} \nu_{e}\right) /\right.$ $\left.\bar{\Gamma}\left(D \rightarrow X e^{+} \nu_{e}\right)\right]$ can be obtained. Current data give $\Gamma\left(\Lambda_{c}^{+} \rightarrow X e^{+} \nu_{e}\right) / \bar{\Gamma}\left(D \rightarrow X e^{+} \nu_{e}\right)=1.44 \pm 0.54$ [7,8]. This ratio is predicted to be $1.67[8,9]$ using an effective-quark theory calculation and about 1.2 based on a calculation using the heavy-quark expansion [10]. Therefore, a more

Published by the American Physical Society under the terms of the Creative Commons Attribution 4.0 International license. Further distribution of this work must maintain attribution to the author(s) and the published article's title, journal citation, and DOI. Funded by SCOAP. precise measurement of $\mathcal{B}\left(\Lambda_{c}^{+} \rightarrow X e^{+} \nu_{e}\right)$ is desirable to test these theoretical predictions.

Measurement of $\mathcal{B}\left(\Lambda_{c}^{+} \rightarrow X e^{+} \nu_{e}\right)$ was only performed by the MARK II collaboration in 1982, with a result of $(4.5 \pm 1.7) \%$ [11] using an $e^{+} e^{-}$collision data sample taken at center-of-mass energies from 4.5 to $6.8 \mathrm{GeV}$. The determination of $\mathcal{B}\left(\Lambda_{c}^{+} \rightarrow X e^{+} \nu_{e}\right)$ is obtained from signal events containing $\bar{\Lambda} e^{+}\left(\bar{p} e^{+}\right)$[12], with the observed $\bar{\Lambda}(\bar{p})$ serving as a tag for a charmed baryon event. All these events are assumed to be from charmed baryon pair production and subsequent $\Lambda_{c}^{+}$semileptonic decay. This assumption should be questioned as there are $e^{+} e^{-} \rightarrow$ $c \bar{c} \rightarrow \bar{D} \bar{p} \Lambda_{c}^{+} X$ continuum events [13]. Besides, they need to estimate the total number of produced charmed baryon events, which has large uncertainties and is model dependent [1]. In this Letter, we present the first absolute measurement of $\mathcal{B}\left(\Lambda_{c}^{+} \rightarrow X e^{+} \nu_{e}\right)$ by employing a double-tag technique [14]. This technique takes advantage of a clean $\Lambda_{c}^{+} \bar{\Lambda}_{c}^{-}$sample just above the threshold and, thus, obviates the need to make the above assumption or estimate the total number of produced charmed baryon events.

Our measurement is performed by analyzing an $e^{+} e^{-}$ collision data sample of $567 \mathrm{pb}^{-1}$ accumulated at $\sqrt{s}=$ 4.6 GeV and recorded with the BESIII detector [15] at the Beijing Electron-Positron Collider II (BEPCII) [16]. A detailed description of the BESIII detector can be found in Ref. [15].

A GEANT4-based [17] Monte Carlo (MC) simulation is used to estimate the signal efficiency, optimize the selection criteria, and understand the backgrounds. In the simulation, the effects of beam-energy spread and initial state radiation (ISR) are incorporated using KKMC [18], and the final-state 
radiation (FSR) is modeled by Pнотоs [19]. A MC sample consisting of $\Lambda_{c}^{+} \bar{\Lambda}_{c}^{-}$pairs, $D_{(s)}^{(*)} \bar{D}_{(s)}^{(*)}$ pairs, ISR to lowermass charmonium $(\psi)$ states, and continuum processes incorporates most expected channels. The known decay modes are modeled with EVTGEN $[20,21]$ using branching fractions taken from the Particle Data Group (PDG) [7], and the remaining unknown decays from the charmonium states with LUNDCHARM [22].

A double-tag technique, first developed by the MARK III collaboration [23], is employed. First, we fully reconstruct one $\bar{\Lambda}_{c}^{-}$and, then, search for candidates of the signal decay in the rest of the event that is recoiling against the tagged $\bar{\Lambda}_{c}^{-}$. Hence, the absolute branching fraction of the inclusive semileptonic decay can be measured without knowing the total number of $\Lambda_{c}^{+} \bar{\Lambda}_{c}^{-}$pairs produced, thus, eliminating the related systematic uncertainty. The tag candidates are reconstructed through the decays $\bar{\Lambda}_{c}^{-} \rightarrow$ $\bar{p} K_{S}^{0}$ and $\bar{\Lambda}_{c}^{-} \rightarrow \bar{p} K^{+} \pi^{-}$, which have large branching fractions and low backgrounds.

The charged tracks, except those from $K_{S}^{0}$, are required to have a polar angle $\theta$ with respect to the beam direction within the multilayer drift chamber (MDC) acceptance $|\cos \theta|<0.93$, and a distance of closest approach to the interaction point (IP) within $10 \mathrm{~cm}$ along the beam direction and $1 \mathrm{~cm}$ in the plane transverse to the beam direction. Particle identification (PID) for charged pions, kaons, and protons is performed by exploiting time-offlight (TOF) information and specific ionization energy loss $d E / d x$ measured by the MDC. The confidence level (C.L.) under each particle hypothesis $(p, K$, or $\pi)$ is calculated; each charged track is assigned the particle type with the largest PID C. L. The $K_{S}^{0}$ meson candidates are reconstructed from two oppositely charged tracks to which no PID criteria are applied and which are assigned the pion mass hypothesis. The charged tracks from the $K_{S}^{0}$ candidate must satisfy $|\cos \theta|<0.93$. Furthermore, due to the long lifetime of the $K_{S}^{0}$ meson, there is a less stringent criterion on the distance of closest approach to the IP in the beam direction of less than $20 \mathrm{~cm}$, and there is no requirement on the distance of closest approach in the plane transverse to the beam direction. The invariant mass of the track pair is required to be in the range $(0.487,0.511) \mathrm{GeV} / c^{2}$. Furthermore, the $\pi^{+} \pi^{-}$pair is constrained to be consistent with originating from a common decay vertex by means of a vertex fit. In addition, the decay length, which is the distance between the IP and the decay vertex, is required to be larger than twice its resolution.

To suppress combinatorial backgrounds, two kinematic variables are used to select the tag candidates. These are the energy difference $\Delta E \equiv E_{\bar{\Lambda}_{c}^{-}}-E_{\text {beam }}$ and the beamconstrained (BC) mass $M_{\mathrm{BC}} \equiv \sqrt{E_{\text {beam }}^{2} / c^{4}-\left|\vec{p}_{\bar{\Lambda}_{c}^{-}}\right|^{2} / c^{2}}$, where $E_{\text {beam }}$ is the beam energy, $E_{\bar{\Lambda}_{c}^{-}}$and $\vec{p}_{\bar{\Lambda}_{c}^{-}}$are the reconstructed energy and three momentum of the tag
TABLE I. Summary of $\Delta E$ requirements, detection efficiencies, and tag yields for the different tag modes.

\begin{tabular}{lccc}
\hline \hline Tag mode & $\Delta E(\mathrm{MeV})$ & Efficiency $(\%)$ & Yield \\
\hline $\bar{\Lambda}_{c}^{-} \rightarrow \bar{p} K_{S}^{0}$ & $(-21,19)$ & $56.5 \pm 0.3$ & $1214 \pm 36$ \\
$\bar{\Lambda}_{c}^{-} \rightarrow \bar{p} K^{+} \pi^{-}$ & $(-20,16)$ & $50.1 \pm 0.1$ & $6092 \pm 82$ \\
\hline \hline
\end{tabular}

candidate in the rest frame of the $e^{+} e^{-}$system, respectively. We require $\Delta E$ to be within $(-3 \sigma, 3 \sigma)$ of the peak of the $\Delta E$ distribution, where $\sigma$ is the resolution of the $\Delta E$ distribution. Table I gives the $\Delta E$ requirements for each tag mode. If there are multiple candidates for the same tag mode in a given event, only the combination with the smallest $|\Delta E|$ is retained for further analysis. To determine the tag yields, we apply a fit to the $M_{\mathrm{BC}}$ distributions, as shown in Fig. 1 . In the fits, the signal shape is modeled by the shape derived from MC simulation convolved with a Gaussian function that describes the resolution difference between data and MC simulation; the combinatorial background is described by an ARGUS function [24]. We obtain the tag yields by subtracting the integral of the background function in the signal region $2.282<M_{\mathrm{BC}}<2.300 \mathrm{GeV} / c^{2}$ from the total number of events in the same region. The tails of the $M_{\mathrm{BC}}$ distribution above the nominal $\Lambda_{c}^{+}$mass are due to the effects of ISR and FSR. The tag yields and the corresponding detection efficiencies are summarized in Table I.

In the selected tag sample of $\bar{\Lambda}_{c}^{-}$candidates, we search for charged tracks consistent with being an electron or positron. To ensure that the charged tracks originate from the IP, the same distance of closest approach selection criteria are used as for the non- $K_{S}^{0}$ daughters of the tag candidates. The track is required to satisfy $|\cos \theta|<0.8$ to ensure that it lies within the acceptance of the barrel of the electromagnetic calorimeter (EMC), which has better energy resolution than the EMC end caps. The momentum of the charged track is required to be greater than $200 \mathrm{MeV} / c$, as it is difficult to separate positrons from other particles with low momenta. The selected tracks are divided into right-sign and wrong-sign samples, where the

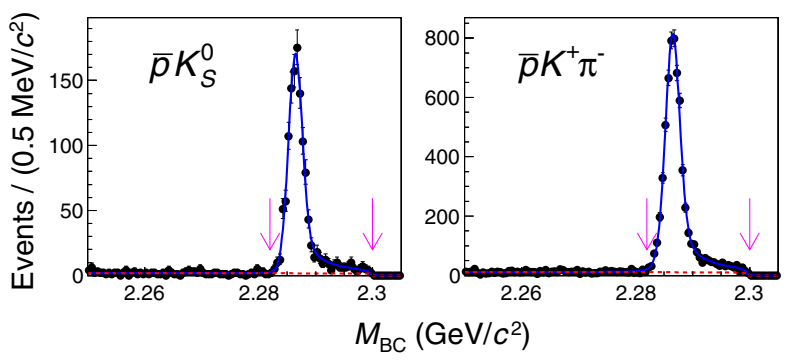

FIG. 1. $M_{\mathrm{BC}}$ distributions for the different tag modes in data. The solid blue line is the total fit, the dashed red line is the background component, and the pink arrows denote the $M_{\mathrm{BC}}$ signal region. 
charge of the right-sign (wrong-sign) track is required to be opposite (equal) to that of the tag candidate.

The PID of the selected tracks is implemented with the information of the $d E / d x$, TOF and EMC, and the C.L. under each particle hypothesis $(e, \pi, K$, or $p)$ is calculated. Positron candidates must satisfy C.L. $(e)>0.001$ and C.L. $(e) /[$ C.L. $(e)+$ C.L. $(\pi)+$ C.L. $(K)+$ C.L. $(p)]>0.8$. To further suppress the backgrounds from charged pions, $E_{e} / p_{e}>0.8$ is required, where $E_{e}$ and $p_{e}$ are the deposited energy in the EMC and momentum measured by the MDC, respectively. The remaining selected charged tracks are assigned the hadron type corresponding to the highest C.L. that is greater than 0.001 . The track is rejected if it does not have a C.L. greater than 0.001 for any hypothesis.

The identified positron sample contains sizable backgrounds from misidentified hadrons. To evaluate these backgrounds, knowledge of their yields and corresponding misidentification probabilities is required. The real rightsign and wrong-sign positron yields are determined individually by unfolding the matrix [25-27]

$$
\left(\begin{array}{l}
N_{e}^{\mathrm{obs}} \\
N_{\pi}^{\mathrm{obs}} \\
N_{K}^{\mathrm{obs}} \\
N_{p}^{\mathrm{obs}}
\end{array}\right)=\left(\begin{array}{llll}
P_{e \rightarrow e} & P_{\pi \rightarrow e} & P_{K \rightarrow e} & P_{p \rightarrow e} \\
P_{e \rightarrow \pi} & P_{\pi \rightarrow \pi} & P_{K \rightarrow \pi} & P_{p \rightarrow \pi} \\
P_{e \rightarrow K} & P_{\pi \rightarrow K} & P_{K \rightarrow K} & P_{p \rightarrow K} \\
P_{e \rightarrow p} & P_{\pi \rightarrow p} & P_{K \rightarrow p} & P_{p \rightarrow p}
\end{array}\right)\left(\begin{array}{c}
N_{e}^{\text {true }} \\
N_{\pi}^{\text {true }} \\
N_{K}^{\text {true }} \\
N_{p}^{\text {true }}
\end{array}\right),
$$

where $N_{a}^{\text {obs }}$ is the observed yield of particle species $a$ ( $a$ denotes $e, \pi, K$, or $p$ ), $P_{a \rightarrow b}$ is the probability of identifying particle $a$ as particle $b$, and $N_{a}^{\text {true }}$ is the true yield of particle $a$ in the studied sample. The elements of the PID efficiency matrix $P_{a \rightarrow b}$ are obtained by studying corresponding control samples selected from data. The charged pion and proton samples are selected from $J / \psi \rightarrow$ $p \bar{p} \pi^{+} \pi^{-}$events. The charged kaon and positron samples are selected from $J / \psi \rightarrow K^{+} K^{-} K^{+} K^{-}$and radiative Bhabha events, respectively. Because of the different event topologies, the PID efficiency of positrons from $\Lambda_{c}^{+} \bar{\Lambda}_{c}^{-}$ pairs (one positron and several hadrons) differs from that from radiative Bhabha scattering events (one electron, one positron, and one shower). The relative difference ( $4.2 \%)$ is corrected by comparing the positron efficiency obtained from radiative Bhabha MC samples and $\Lambda_{c}^{+} \bar{\Lambda}_{c}^{-}$pair $\mathrm{MC}$ samples. No correction to the other elements is implemented. The momentum dependence of the PID efficiency matrix is mostly determined in intervals of $100 \mathrm{MeV} / c$, though some intervals are wider due to limited statistics, as presented in Fig. 2. The muon component is omitted in the unfolding procedure due to its small yields (almost the same as the positron yields), the small mis-PID probability from muon to positron (similar to that from pion to positron, shown in Fig. 2) and the negligible effect on the branching fraction measurement. In addition, because the selected pion sample contains the muon component

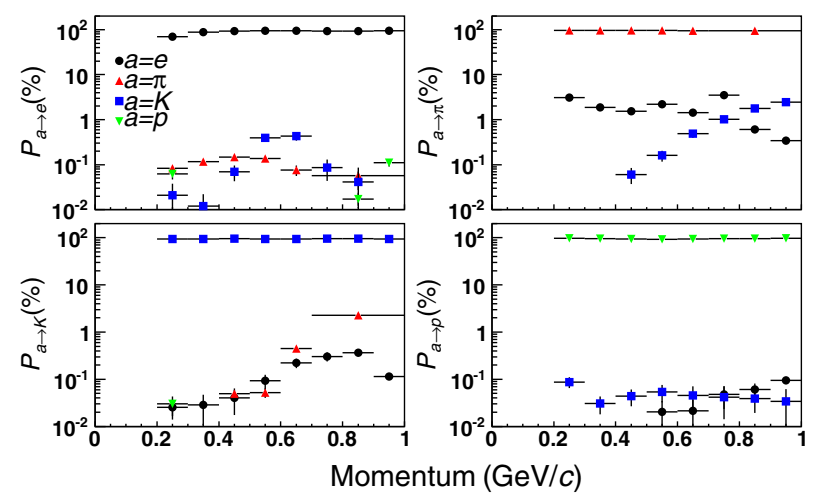

FIG. 2. PID efficiencies obtained from data.

due to their similar PID behavior in the BESIII detector, the muon component is implicitly taken into account.

To estimate the contribution from non- $\Lambda_{c}^{+}$decays in the signal region, the unfolded positron yield in the $M_{\mathrm{BC}}$ sideband region is scaled by a factor of 0.78 that accounts for the relative amount of background in the sideband and signal regions determined by the fit to the $M_{\mathrm{BC}}$ distribution. Since low-background tag modes are used, the contribution from non- $\Lambda_{c}^{+}$decays is small (3.8\%).

The right-sign sample contains primary positrons, which directly originate from $\Lambda_{c}^{+}$decays, and secondary positrons, not directly arising from $\Lambda_{c}^{+}$decays and originating predominantly from $\gamma$ conversions and $\pi^{0}$ Dalitz decays. Detailed MC studies indicate that the secondary positrons are charge symmetric; hence, their yield can be evaluated from the wrong-sign positron sample and subtracted from the total right-sign positron yields. The reliability of the wrong-sign subtraction has been validated by MC studies.

The tracking efficiency in a given momentum interval, including geometrical acceptance $(80 \%$ due to the cut of $|\cos \theta|<0.8$ ), track reconstruction efficiency, selection efficiency, and resolution effects, is corrected by unfolding the following matrix equation:

$$
N_{i}^{\text {true }}=\sum_{j} T(i \mid j) N_{j}^{\mathrm{pro}},
$$

where the tracking efficiency matrix $T(i \mid j)$ describes the probability of positrons produced in the $j$ th momentum interval to be reconstructed in the $i$ th momentum interval, $N_{j}^{\mathrm{pro}}$ is the number of primary positrons produced in the $j$ th momentum interval, and $N_{i}^{\text {true }}$ is the true yield of positron reconstructed in the $i$ th momentum interval. The tracking efficiency matrix is obtained by studying the positron MC sample selected from $\Lambda_{c}^{+}$semileptonic events. After this procedure is applied, we obtain the efficiency-corrected positron momentum spectrum above $200 \mathrm{MeV} / c$ in the laboratory frame. Table II summarizes the positron yields obtained after each correction step.

The fraction of positrons below $200 \mathrm{MeV} / c$ is obtained by fitting the efficiency-corrected positron momentum 
TABLE II. Positron yields in data after each procedure. The uncertainties are statistical.

\begin{tabular}{lcr}
\hline \hline$\Lambda_{c}^{+} \rightarrow X e^{+} \nu_{e}$ & Right sign & Wrong sign \\
\hline Observed yields & & \\
$\quad$ Tag signal region & $228.0 \pm 15.1$ & $26.0 \pm 5.1$ \\
$\quad$ Tag sideband region & $11.0 \pm 3.3$ & $2.0 \pm 1.4$ \\
PID unfolding & & \\
$\quad$ Tag signal region & $250.1 \pm 17.1$ & $28.3 \pm 6.2$ \\
$\quad$ Tag sideband region & $12.1 \pm 3.8$ & $1.7 \pm 1.5$ \\
Sideband subtraction & $240.7 \pm 17.4$ & $27.0 \pm 6.3$ \\
Wrong-sign subtraction & $213.7 \pm 18.5$ & \\
Correction of tracking efficiency & $272.1 \pm 23.5$ & \\
\hline \hline
\end{tabular}

spectrum with the sum of the spectra of the exclusive decay channels (Table III), as shown in Fig. 3. In the fit, the branching fraction of each component is allowed to vary within the given uncertainty. From the fit, we obtain the fraction of positrons below $200 \mathrm{MeV} / c$ to be $(5.6 \pm 1.5) \%$, where the uncertainty is systematic derived from variations of the fit assumptions. The branching fraction of the inclusive semileptonic decay of the $\Lambda_{c}^{+}$baryon is then calculated with

$\mathcal{B}\left(\Lambda_{c}^{+} \rightarrow X e^{+} \nu_{e}\right)=\frac{N^{\mathrm{pro}}\left(p_{e}>200 \mathrm{MeV} / c\right)}{N_{\mathrm{tag}}\left[1-f\left(p_{e}<200 \mathrm{MeV} / c\right)\right]}$,

where $N^{\text {pro }}\left(p_{e}>200 \mathrm{MeV} / c\right)$ is the yield of positrons with momentum $p_{e}$ above $200 \mathrm{MeV} / c$ after the correction of the tracking efficiency, $N_{\text {tag }}$ is the tag yield, and $f\left(p_{e}<200 \mathrm{MeV} / c\right)$ is the fraction of positrons below $200 \mathrm{MeV} / c$. Finally, we obtain $\mathcal{B}\left(\Lambda_{c}^{+} \rightarrow X e^{+} \nu_{e}\right)=$ $(3.95 \pm 0.34) \%$, where the uncertainty is statistical only.

The systematic uncertainties in this analysis are listed in Table IV. The tag yield systematic uncertainty is estimated to be $1.0 \%$ by using alternative fits to the $M_{\mathrm{BC}}$ distribution with different signal shapes, background parameters, and fitting ranges. The systematic uncertainty related to

TABLE III. $\Lambda_{c}^{+}$semileptonic decays used to extrapolate the positron momentum spectrum. The branching fraction of the $\Lambda_{c}^{+} \rightarrow \Lambda e^{+} \nu_{e}$ decay is from the BESIII measurement [5] and the uncertainty of the unobserved decay channels is $100 \%$ of the predicted branching fractions. The form factor of the $\Lambda_{c}^{+} \rightarrow$ $\Lambda e^{+} \nu_{e}$ decay is taken from QCD sum rules [28] and the other two, unobserved, semileptonic decay modes are generated by PYTHIA [29] according to the simple $V-A$ matrix element.

\begin{tabular}{lcc}
\hline \hline Decay channel & $\mathcal{B}(\%)$ & Model \\
\hline$\Lambda_{c}^{+} \rightarrow \Lambda e^{+} \nu_{e}$ & $3.63 \pm 0.43[5]$ & $F_{1}^{V}\left(q^{2}\right)$ \\
& & $=2.52 / 5.09-q^{2}[28]$ \\
$\Lambda_{c}^{+} \rightarrow \Lambda(1405) e^{+} \nu_{e}$ & $0.38 \pm 0.38[30]$ & PYTHIA [29] \\
$\Lambda_{c}^{+} \rightarrow n e^{+} \nu_{e}$ & $0.27 \pm 0.27[31]$ & PYTHIA [29] \\
\hline \hline
\end{tabular}

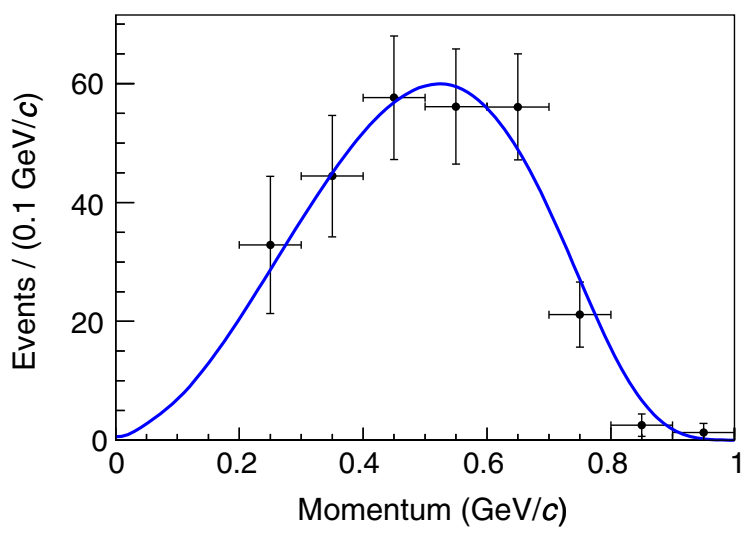

FIG. 3. Extrapolation of the positron momentum spectrum in the laboratory frame obtained from data, shown as points with error bars. The blue curve shows the extrapolated spectrum.

the tracking efficiency is estimated to be $1.0 \%$ by studying radiative Bhabha scattering events [5]. The systematic uncertainty in the positron identification efficiency is estimated by comparing the positron PID efficiencies in different $\mathrm{MC}$ simulated semileptonic $\Lambda_{c}^{+}$decays. The largest relative difference of the positron PID efficiency is assigned as the systematic uncertainty. The uncertainties in the other elements of the PID efficiency matrix are estimated by comparing the matrix elements obtained from $\Lambda_{c}^{+} \bar{\Lambda}_{c}^{-}$pair MC samples with those obtained from MC samples of radiative Bhabha events, $J / \psi \rightarrow p \bar{p} \pi^{+} \pi^{-}$and $J / \psi \rightarrow K^{+} K^{-} K^{+} K^{-}$MC samples. Adding them in quadrature, we assign $0.9 \%$ as the systematic uncertainty related to PID. The uncertainty associated with the $M_{\mathrm{BC}}$ sideband subtraction is estimated to be $0.5 \%$ by using an alternative $M_{\mathrm{BC}}$ sideband region. To estimate the uncertainty in the extrapolation of the positron momentum spectrum, we perform an alternative fit in which the branching fraction of each fit component is unconstrained. In addition, we use an alternative form-factor model and repeat the fit. Adding these effects in quadrature, we attribute $1.5 \%$ as the systematic uncertainty related to the extrapolation procedure. The uncertainty due to limited statistics of data and MC simulation used to determine the PID efficiency matrix and tracking efficiency matrix is estimated by repeating the PID unfolding procedure and correction of tracking

TABLE IV. Sources of systematic uncertainties.

\begin{tabular}{lc}
\hline \hline Source & Relative uncertainty $(\%)$ \\
\hline Tag yield & 1.0 \\
Tracking & 1.0 \\
PID & 0.9 \\
Sideband subtraction & 0.5 \\
Extrapolation & 1.5 \\
Data and MC statistics & 0.4 \\
Sum & 2.3 \\
\hline \hline
\end{tabular}


TABLE V. Comparison of the branching fraction (in $10^{-2}$ ) and ratio of the semileptonic decay width between experimental measurements and theoretical predictions.

\begin{tabular}{lcc}
\hline \hline & & {$\left[\Gamma\left(\Lambda_{c}^{+} \rightarrow X e^{+} \nu_{e}\right) /\right.$} \\
Result & $\Lambda_{c}^{+} \rightarrow X e^{+} \nu_{e}$ & $\left.\bar{\Gamma}\left(D \rightarrow X e^{+} \nu_{e}\right)\right]$ \\
\hline BESIII & $3.95 \pm 0.35$ & $1.26 \pm 0.12$ \\
MARK II [11] & $4.5 \pm 1.7$ & $1.44 \pm 0.54$ \\
Effective-quark method [8,9] & & 1.67 \\
Heavy-quark expansion [10] & & 1.2 \\
\hline \hline
\end{tabular}

efficiency. In each repetition, we vary each element of the PID efficiency matrix and tracking efficiency matrix within the corresponding error simultaneously. The corresponding systematic uncertainty is derived from 10000 independent repetitions and is estimated to be $0.4 \%$. Adding all uncertainties in quadrature, the total systematic uncertainty is determined to be $2.3 \%$.

The absolute branching fraction of the inclusive semileptonic decays of the $\Lambda_{c}^{+}$baryon is determined to be $\mathcal{B}\left(\Lambda_{c}^{+} \rightarrow X e^{+} \nu_{e}\right)=(3.95 \pm 0.34 \pm 0.09) \%$, where the first and second uncertainties are statistical and systematic, respectively. Compared with the branching fraction of $\Lambda_{c}^{+} \rightarrow \Lambda e^{+} \nu_{e}$ measured by the BESIII collaboration [5], the ratio $\left[\mathcal{B}\left(\Lambda_{c}^{+} \rightarrow \Lambda e^{+} \nu_{e}\right) / \mathcal{B}\left(\Lambda_{c}^{+} \rightarrow X e^{+} \nu_{e}\right)\right]$ is determined to be $(91.9 \pm 12.5 \pm 5.4) \%$, where the systematic uncertainty related to the tracking efficiency of the positron cancels. Using the known $\Lambda_{c}^{+}$lifetime [7], we obtain the semileptonic decay width $\Gamma\left(\Lambda_{c}^{+} \rightarrow X e^{+} \nu_{e}\right)=(1.98 \pm$ $0.18) \times 10^{11} \mathrm{~s}^{-1}$. Comparing this with the charge-averaged semileptonic decay width of nonstrange charmed mesons $\bar{\Gamma}\left(D \rightarrow X e^{+} \nu_{e}\right)[7]$, the ratio $\left[\Gamma\left(\Lambda_{c}^{+} \rightarrow X e^{+} \nu_{e}\right) / \bar{\Gamma}(D \rightarrow\right.$ $\left.\left.X e^{+} \nu_{e}\right)\right]$ is determined to be $1.26 \pm 0.12$. A comparison of the branching fraction and ratio of the semileptonic decay width between experimental measurements and theoretical predictions can be found in Table V.

In summary, by analyzing a data sample corresponding to an integrated luminosity of $567 \mathrm{pb}^{-1}$ taken at a center-ofmass energy $\sqrt{s}=4.6 \mathrm{GeV}$, we report the absolute measurement of the inclusive semileptonic $\Lambda_{c}^{+}$decay branching fraction $\mathcal{B}\left(\Lambda_{c}^{+} \rightarrow X e^{+} \nu_{e}\right)=(3.95 \pm 0.34 \pm 0.09) \%$. The uncertainty is reduced by a factor of 4 compared to the MARK II result [11]. Based on the BESIII measurements [5], we obtain the ratio of the branching fraction to be $\left[\mathcal{B}\left(\Lambda_{c}^{+} \rightarrow \Lambda e^{+} \nu_{e}\right) / \mathcal{B}\left(\Lambda_{c}^{+} \rightarrow X e^{+} \nu_{e}\right)\right]=(91.9 \pm 12.5 \pm 5.4) \%$. We also determine the ratio $\left[\Gamma\left(\Lambda_{c}^{+} \rightarrow X e^{+} \nu_{e}\right) / \bar{\Gamma}(D \rightarrow\right.$ $\left.\left.X e^{+} \nu_{e}\right)\right]=1.26 \pm 0.12$, which restricts different models as given in Table $\mathrm{V}$.

The BESIII collaboration thanks the staff of BEPCII and the IHEP computing center for their strong support. This work is supported in part by the National Key Basic Research Program of China under Contract No. 2015CB856700; the National Natural Science Foundation of China (NSFC) under Contracts
No. 11235011, No. 11335008, No. 11425524, No. 11625523, and No. 11635010; the Chinese Academy of Sciences (CAS) Large-Scale Scientific Facility Program; the CAS Center for Excellence in Particle Physics (CCEPP); Joint Large-Scale Scientific Facility Funds of the NSFC and CAS under Contracts No. U1332201, No. U1532257, and No. U1532258; CAS under Contracts No. KJCX2-YW-N29 and No. KJCX2YW-N45; CAS Key Research Program of Frontier Sciences under Contracts No. QYZDJ-SSW-SLH003 and No. QYZDJ-SSW-SLH040; 100 Talents Program of CAS; National 1000 Talents Program of China; INPAC and Shanghai Key Laboratory for Particle Physics and Cosmology; German Research Foundation DFG under Contracts No. Collaborative Research Center CRC 1044 and No. FOR 2359; Istituto Nazionale di Fisica Nucleare, Italy; Koninklijke Nederlandse Akademie van Wetenschappen (KNAW) under Contract No. 5304CDP03; Ministry of Development of Turkey under Contract No. DPT2006K-120470; National Science and Technology fund; The Swedish Research Council; U.S. Department of Energy under Contracts No. DE-FG0205ER41374, No. DE-SC-0010118, No. DE-SC-0010504 and No. DE-SC-0012069; University of Groningen (RuG) and the Helmholtzzentrum fuer Schwerionenforschung GmbH (GSI), Darmstadt; WCU Program of National Research Foundation of Korea under Contract No. R322008-000-10155-0.

\footnotetext{
${ }^{a} A 1 s o$ at Bogazici University, 34342 Istanbul, Turkey.

${ }^{\mathrm{b}}$ Also at the Moscow Institute of Physics and Technology, Moscow 141700, Russia.

${ }^{\mathrm{c}}$ Also at the Functional Electronics Laboratory, Tomsk State University, Tomsk 634050, Russia.

dAlso at the Novosibirsk State University, Novosibirsk 630090, Russia.

"Also at the NRC "Kurchatov Institute," PNPI, Gatchina 188300, Russia.

${ }^{\mathrm{f}}$ Also at Istanbul Arel University, 34295 Istanbul, Turkey.

${ }^{\mathrm{g}}$ Also at Goethe University Frankfurt, 60323 Frankfurt am Main, Germany.

${ }^{\mathrm{h}}$ Also at Key Laboratory for Particle Physics, Astrophysics and Cosmology, Ministry of Education; Shanghai Key Laboratory for Particle Physics and Cosmology; Institute of Nuclear and Particle Physics, Shanghai 200240, People's Republic of China.

${ }^{\mathrm{i}}$ Government College Women University, Sialkot-51310 Punjab, Pakistan.

${ }^{j}$ Present address: Center for Underground Physics, Institute for Basic Science, Daejeon 34126, Korea.

[1] G. S. Abrams et al. (MARK II Collaboration), Phys. Rev. Lett. 44, 10 (1980).

[2] M. Gronau and J. L. Rosner, Phys. Rev. D 97, 116015 (2018).

[3] H. Albrecht et al. (ARGUS Collaboration), Phys. Lett. B 269, 234 (1991).
} 
[4] T. Bergfeld et al. (CLEO Collaboration), Phys. Lett. B 323, 219 (1994).

[5] M. Ablikim et al. (BESIII Collaboration), Phys. Rev. Lett. 115, 221805 (2015).

[6] M. Ablikim et al. (BESIII Collaboration), Phys. Lett. B 767, 42 (2017).

[7] C. Patrignani et al. (Particle Data Group), Chin. Phys. C 40, 100001 (2016).

[8] M. Gronau and J. L. Rosner, Phys. Rev. D 83, 034025 (2011).

[9] J. L. Rosner, Phys. Rev. D 86, 014017 (2012).

[10] A. V. Manohar and M. B. Wise, Phys. Rev. D 49, 1310 (1994).

[11] E. Vella et al. (MARK II Collaboration), Phys. Rev. Lett. 48, 1515 (1982).

[12] In MARK II measurement, events containing a $p$ and not a $\bar{p}$ are excluded to reduce the background from beam-gas interactions.

[13] D. E. Jaffe et al. (CLEO Collaboration), Phys. Rev. D 62, 072005 (2000).

[14] Throughout this Letter, charge-conjugate modes are implied unless otherwise stated.

[15] M. Ablikim et al. (BESIII Collaboration), Nucl. Instrum. Methods Phys. Res., Sect. A 614, 345 (2010).

[16] C. H. Yu et al., Proceedings of the IPAC2016, Busan, Korea, 2016 (JACoW, Geneva, 2016).

[17] S. Agostinelli et al. (GEANT4 Collaboration), Nucl. Instrum. Methods Phys. Res., Sect. A 506, 250 (2003).
[18] S. Jadach, B. F. L. Ward, and Z. Wąs, Phys. Rev. D 63, 113009 (2001).

[19] E. Richter-Was, Phys. Lett. B 303, 163 (1993).

[20] R. G. Ping, Chin. Phys. C 32, 599 (2008).

[21] D. J. Lange, Nucl. Instrum. Methods Phys. Res., Sect. A 462, 152 (2001).

[22] J. C. Chen, G. S. Huang, X. R. Qi, D. H. Zhang, and Y. S. Zhu, Phys. Rev. D 62, 034003 (2000).

[23] R. M. Baltrusaitis et al. (MARK III Collaboration), Phys. Rev. Lett. 56, 2140 (1986).

[24] H. Albrecht et al. (ARGUS Collaboration), Phys. Lett. B 241, 278 (1990).

[25] A. Hocker and V. Kartvelishvili, Nucl. Instrum. Methods Phys. Res., Sect. A 372, 469 (1996).

[26] N. E. Adam et al. (CLEO Collaboration), Phys. Rev. Lett. 97, 251801 (2006).

[27] D. M. Asner et al. (CLEO Collaboration), Phys. Rev. D 81, 052007 (2010).

[28] R. S. Marques de Carvalho, F. S. Navarra, M. Nielsen, E. Ferreira, and H. G. Dosch, Phys. Rev. D 60, 034009 (1999).

[29] T. Sjostrand, P. Edén, C. Friberg, L. Lönnblad, G. Miu, S. Mrenna, and E. Norrbin, Comput. Phys. Commun. 135, 238 (2001).

[30] M. Pervin, W. Roberts, and S. Capstick, Phys. Rev. C 72, 035201 (2005).

[31] M. A. Ivanov, V. E. Lyubovitskij, J. G. Körner, and P. Kroll, Phys. Rev. D 56, 348 (1997). 